\title{
THE EFFECTS OF A RUNNING CONSISTENCY PROGRAMME ON FOOTFALL VARIABILITY AND PERFORMANCE IN THE LONG JUMP
}

\author{
MARCIN STARZAK ${ }^{1}$, HUBERT MAKARUK ${ }^{1}$, ANNA STARZAK² \\ Józef Piłsudski University of Physical Education in Warsaw, \\ Faculty of Physical Education and Sport in Biata Podlaska², \\ Department of Theory and Technology of training ${ }^{1}$
}

\author{
Mailing address: Marcin Starzak, Faculty of Physical Education and Sport, 2 Akademicka Street, 21-500 Biała Podlaska, \\ tel.: +48 602230467, fax: +48 83 3428800, e-mail: marcin.starzak@awf-bp.edu.pl
}

\begin{abstract}
Introduction. The main purpose of this study was to evaluate the effect of a training programme aimed to enhance toe-toboard consistency on footfall variability and performance in the long jump. Material and methods. The study involved 36 male physical education students. The experimental group participated in a 12-week training programme, whereas the control group was limited to taking part in the classes held at university. All participants performed 6 long jump trials during two testing sessions. The kinematic parameters were assessed using the Optojump Next device and were further analysed to determine the variability of footfall placement during the approach run. Results. The analysis revealed a significant $(\mathrm{p}<0.01)$ decrease in footfall variability in the experimental group between the pre-test and post-test. After the completion of the training programme, the participants significantly $(\mathrm{p}<0.05)$ improved their take-off accuracy. Additionally, they significantly $(\mathrm{p}<0.05)$ increased their velocity in the last five steps before take-off and the effective distance of the jump $(p<0.001)$. Conclusions. The results of this study indicate that through specific training, it is possible to improve the consistency of the steps in the acceleration phase of the approach run in the long jump. Moreover, decreasing footfall variability helps achieve a more stable step pattern which may be beneficial for greater accuracy at the take-off board and makes it possible to increase step velocity at the final stage of the approach run.
\end{abstract}

Key words: athletics, constraints, stability, step pattern

\section{Introduction}

From the dynamical system perspective, we never exhibit the same movement patterns while searching for an optimal solution to a given task. Therefore, every movement of the body leads to different changes in the movement outcome. Movement variability is regarded as a variation in human motor behaviour across multiple repetitions of a task [1]. From the traditional motor learning perspective, movement variability is related to noise in the central nervous system which should be eliminated or removed [2]. In the dynamical approach, variability is interpreted as a mechanism that allows for the stabilisation of action and adaptation to numerous constraints [3]. Recently, it has been considered as an essential element of the motor system which promotes flexibility in adapting to perturbations [4].

In the motor learning process, movement variability can be viewed as searching for a stable and functional state of coordination [5]. It can also be a skill indicator, as the level of variability displayed by performers tends to change with experience. Many studies have reported differences between movement patterns depending on levels of expertise, and they have demonstrated that high-level performers show better consistency and stability of the action. Stergiou et al. [6] generally stated that increased movement variability indicates less cooperative behaviour, while decreased variability is a sign of more stable behaviour. An analysis of movement patterns in different sports and motor skills shows that movement variability decreases with task-specific practice, which can be observed among others when unskilled and skilled athletes are compared. This has been confirmed by studies of variability in many discrete sports actions, for exam- ple in golf swings [7] and in basketball free-throws [8], where this has been attributed to a decrease in selected joint movement variability with expertise. In contrast, some researchers suggest that despite years of practice, athletes are unable to produce invariant movement patterns $[9,10]$. Others suggest that regardless of experience, athletes can display similar levels of variability, but experts can exploit this variability in a functional manner [11, 12]. However, there is still no clear evidence of how this variability changes as a result of the learning process.

This functional variability emerges in the long jump, where the performance of the approach run forces athletes to adapt their movement pattern to different constraints. It was previously well observed that either expert or novice long jumpers displayed an ascending-descending trend of step-to-step variability in relation to the take-off board [13, 14, 15]. For example, an analysis of intra-trial footfall variability showed that jumpers exhibit a two-phase strategy during the approach run [13]. In the first phase, the variability of footfall placement constantly increases (acceleration phase), while in the second phase, accumulated variability systematically decreases (zeroing-in phase) until the take-off board is reached.

These last step adjustments during the approach run constantly interact with different constraints and simultaneously reflect the search for an optimal solution that would allow the athlete to accurately hit the take-off board at the highest possible horizontal velocity. These regulations influence the performance of the jump, which has been observed in the past [16]. For example, Starzak et al. [17] found that different environmental constraints lead to different patterns of step length adjustments. Athletes who jump without the take-off board produce 
a lower level of intra-trial variability and start to regulate their steps earlier in the approach run. This provides an opportunity to control movement better and results in higher footfall precision. Similarly, Theodorou et al. [18] claimed that stronger environmental stimuli allow athletes to regulate their steps earlier and that way focus on developing horizontal velocity and proper take-off technique. Some researchers underline the positive effect of enhancing jumpers' interaction with the environment by introducing additional tools (i.e. coaching check marks) into the execution of the approach run. This seems to be beneficial for lowering footfall variability and for developing consistency in the step pattern [19]. Recently, it has been found that the use of extra external aids promotes better control of the approach run, and it can also be an effective way of decreasing footfall variability [20]. Therefore, it may result in better accuracy at the take-off board. Moreover, Makaruk et al. [21] found that lower levels of footfall variability during long and triple jump approach runs could lead to greater take-off accuracy and significantly decrease the number of foul jumps.

A well-established movement pattern seems to be crucial in the long jump, where the proper execution of the approach run is one of the requirements of a successful jump. However, it is nearly impossible to maintain movement invariance throughout the numerous repetitions, as athletes are influenced by several constraints [9]. Nevertheless, some authors propose practical solutions which may improve the performance of the long jump approach run. Lundin and Berg [22] concluded that the primary goal is to start with improving the acceleration phase through performing repetitions in training using various forms of executing the approach run (i.e. approaching with and without jumping). Others suggest focusing on developing an appropriate running rhythm which could help to improve performance by reducing approach run velocity [23]. For instance, they recommend placing mini-hurdles or different cues at selected distances, which could enable athletes to develop a step pattern and simultaneously increase running speed. Using such tools can be an effective way to establish a desirable step length by setting intentional distances between markers [24]. Another solution could be to make it possible for athletes to learn transition running by implementing check marks at varying distances which show points where the running speed needs to be changed. Based on the perception-action coupling mechanism, individuals would then be forced to control the step length and make constant adjustments to their locomotor pattern.

The above-mentioned findings indicate that the level of footfall placement variability during the execution of the long jump approach run depends on the continuous process of an athlete's interaction with different constraints. On the other hand, developing a more stable step pattern and an appropriate running rhythm seems to be an essential element of an approach run training routine. Therefore, the purpose of this study was to investigate how footfall variability changes during the approach run following the implementation of a training programme aimed to enhance step stability and consistency in non-long jumpers. Moreover, we wanted to find out if these changes in movement variability can influence long jump performance.

\section{Material and methods}

\section{Subjects}

Thirty-six male students volunteered to participate in the study. All subjects were physical education students and were not experienced in any sport activity. They were moderately active because of the nature of their studies, which include a number of classes involving physical activity, such as courses in athletics, gymnastics, and team sports. Their only experience in the long jump was that they had completed a long jump course, so they were considered non-long jumpers. Participants were randomly assigned to either the experimental group (EXP, $\mathrm{n}=$ $20)$ or the control group $(\mathrm{CON}, \mathrm{n}=16)$. Detailed characteristics of the groups are presented in table 1. Subjects from both groups were asked to limit their activity to daily tasks and classes scheduled at university. Apart from that, subjects from the EXP group attended the training programme. All participants were informed about the purpose of the study and gave their written informed consent. The study was approved by the University Ethics Committee.

Table 1. Characteristics of the experimental and control groups

\begin{tabular}{|c|c|c|}
\hline & $\operatorname{EXP}(n=20)$ & $\operatorname{CON}(n=16)$ \\
\hline Age [years] & $20.9 \pm 0.4$ & $21.0 \pm 0.5$ \\
\hline Body height [m] & $1.81 \pm 0.04$ & $1.79 \pm 0.03$ \\
\hline Body mass [kg] & $72.7 \pm 6.6$ & $71.1 \pm 7.1$ \\
\hline
\end{tabular}

$\mathrm{EXP}=$ experimental group; $\mathrm{CON}=$ control group.

\section{Training protocol}

Prior to the training programme, all the subjects form the EXP group performed a training session that included runs over wooden slats ( $1 \mathrm{~m}$ in length; $5 \mathrm{~cm}$ in width; $2 \mathrm{~cm}$ in height) to estimate the individual spacing between them. Afterward, they were divided into the two separate groups, according to the individual distances used during the training session. The distance between the slats was 1.9 metres and 2 metres for the first and second group, respectively.

The experimental training programme consisted of 12 practice weeks, with 2 training sessions held per week. Each training session was preceded by a 15-minute dynamic warm-up. The warm-up routine included a 5-minute low-intensity run followed by basic stretching exercises which were aimed to improve the suppleness of all major groups of muscles (i.e. lunge walks, sidewalks, butt kicks, and high knees) and completed with 30-metre submaximal runs. A detailed description of the training programme is presented in table 2 . The distance of each of the running exercises was approximately similar to the distance of a participant's approach runs. The training exercises are illustrated in figure 1 .

\section{Apparatus and measurements}

The Optojump Next (Microgate, Italy) measurement system was used to evaluate the kinematic parameters during the testing sessions. This device consists of two 1-metre-long units (one receiving unit and one transmitting unit) placed parallel with each other at a height of $3 \mathrm{~mm}$ above the floor. For the purpose of this study, a 25-metre path arranged outside the lines along the runway was used. The Optojump Next provides accurate information concerning step length and the position of the foot during the approach run. A pilot study was conducted to assess the reliability of the selected variables. The intra-class correlation coefficients were strong for all the parameters tested (ICCs $=0.90-0.96)$.

The pre-test session took place one week before the start of the experimental programme, and the post-test session was conducted within one week after the completion of the programme. Every training session and both testing sessions took 
Table 2. Training programme of the experimental group

Weeks 1-3

High knees over slats + transition to running (Slats in the first $10 \mathrm{~m}$ ) Acceleration runs (Fast - Slow - Fast)

$4 \times 30 m(10 m+20 m)$

$4 \times 30 m(10 m-10 m-10 m)$

\section{Weeks 4-6}

High knees over slats + transition to running (Slats in the first $10 \mathrm{~m}$ ) Acceleration runs (Fast - Slow - Fast)*

Runs over slats

$4 \times 30 m(10 m+20 m)$

$3 \times 30 m(10 m-10 m-10 m)$

$4 \times 30 \mathrm{~m}$ (slats in the last $5 \mathrm{~m}$ )

\section{Weeks 7-9}

High knees over slats + transition to running (Slats in the first $10 \mathrm{~m}$ ) Acceleration runs (Fast - Slow - Fast) ${ }^{*}$

Runs over slats

$5 \times 30 m(10 m+20 m)$

$3 \times 30 m(10 m-10 m-10 m)$

$5 \times 30 \mathrm{~m}$ (slats in the last $10 \mathrm{~m}$ )

\section{Weeks 10-12}

High knees over slats + transition to running (Slats in the first $10 \mathrm{~m}$ ) Acceleration runs (Fast - Slow - Fast)*

Runs over slats

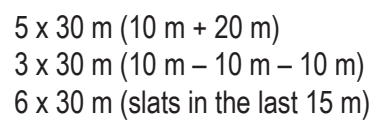

* = check marks placed where pace was changed.

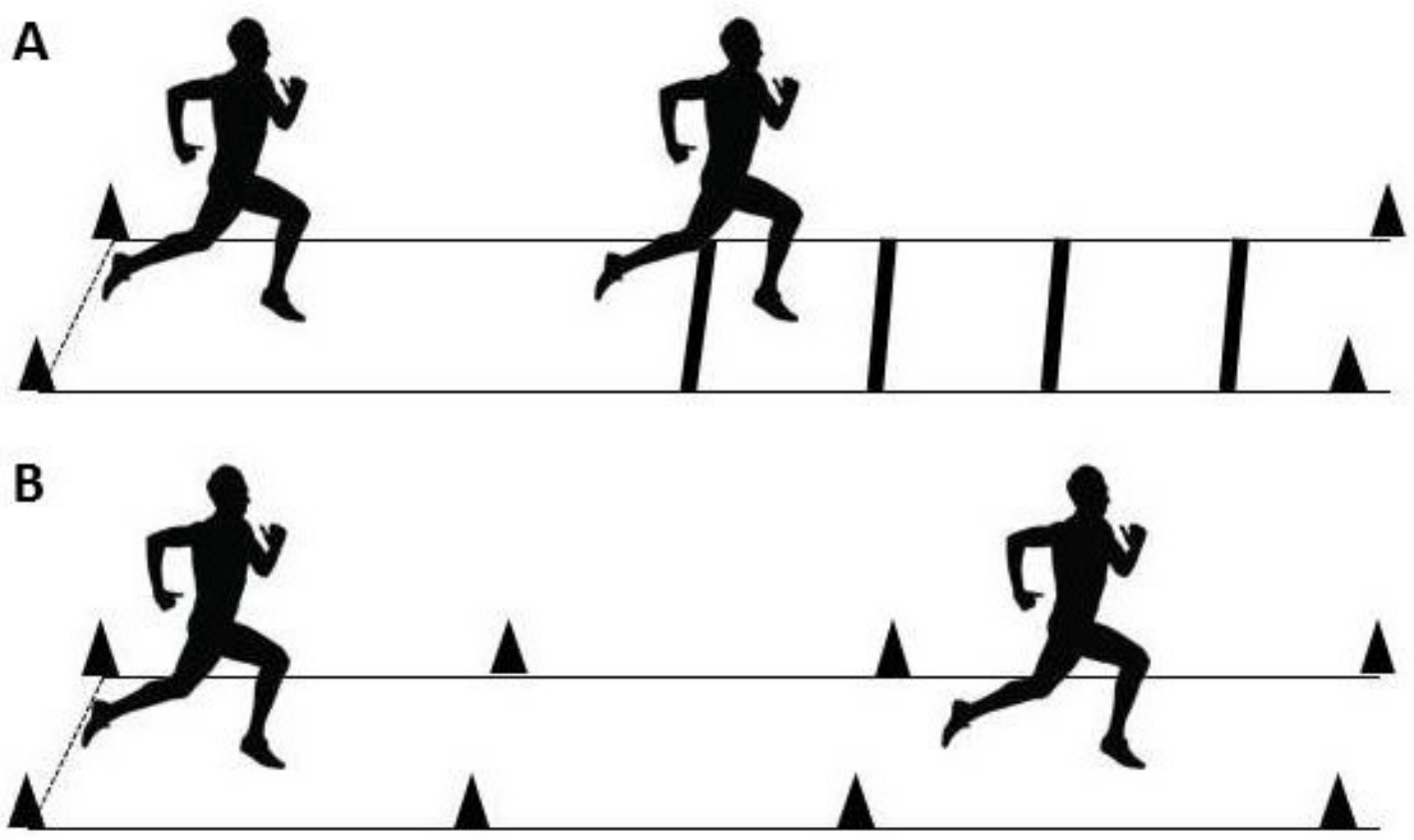

Figure 1. Illustrations of the exercises: A - Runs over slats; B - Acceleration runs (Fast - Slow - Fast)

place at an athletics indoor hall to ensure weather condition stability. Before the start of both testing sessions, the subjects performed an individual 15-minute dynamic warm-up. All participants performed 6 long jump trials according to standard competition rules, except distance measurement. Every jump (including foul jumps) was measured from the toe of the jump- ing foot to the nearest trace left by the subject in the sandpit in order to assess the effective distance of the jump. The breaks between each jump were approximately 7 to 9 minutes long [25]. The length of the approach run was different for each participant and was in the range of 14 to 16 steps $(23.9-27.3 \mathrm{~m})$. All the subjects were asked to begin the run-up from the standing 
position and to keep the same number of steps of the approach run from the pre-test to the post-test session. For the purpose of the study, the final 10 steps (11 support phases) were used for further analysis. A total number of 235 jumps for the EXP group and 188 jumps for the CON group were included in the analysis.

\section{Data analysis}

Assessment of footfall placement variability and step velocity

Step velocity was calculated using the Optojump Next measurement system which determines the average velocity of a single step. This device defines velocity as the relation between two consecutive footfalls and the sum of the first contact time and the flight time.

The toe-to-board distance (TBD) is the horizontal distance between the toe of the subject's support foot and the front edge of the take-off board. The method of measuring TBD used in the current study was previously used to evaluate the variability of footfall placement during the approach run in the long and triple jump [26].

In order to identify movement variability during the approach run, we used the position of the consecutive foot of each participant. The variability of footfall placement was determined by calculating the standard deviation (SD) of the toe-toboard distance across trials.

Percentage distribution of step length adjustment

The percentage distribution of step length adjustment (ADJ\%) was also calculated. According to Hay [15], the formula below makes it possible to determine the percentage of step adjustment between the final steps to total adjustments made by the subject during the approach run.

The formula is:

$$
A D J_{\%}=\frac{\left(S D_{i}-S D_{i-1}\right)}{\left(S D_{\max }-S D_{t o}\right)} \times 100 \%
$$

where $S D$ is the standard deviation of the toe-to-board distance, $i$ is ith-last step (support phase), $S D_{\max }$ is the maximal standard deviation of the toe-to-board distance, and $S D_{\text {to }}$ is the standard deviation of the toe-to-board distance during take-off.

\section{Statistical analysis}

Before the analysis, normality and homogeneity assumptions were checked using the Shapiro-Wilk test. The general linear model (GLM) repeated measures test was employed to compare the following factors: Group (EXP - CON) x Time (pretest - post-test) x Steps $(11-1)$. One-way ANOVA with repeated measures was used to compare the groups for the distance jumped, the accuracy of the take-off, and the maximum toe-toboard values. In all cases, statistically significant results were further analysed using Tukey's HSD post-hoc test. Statistical significance was set at $\mathrm{p}<0.05$. All the statistical calculations were made using Statistica 12.0 PL and Microsoft Excel 2010.

\section{Results}

\section{Variability of footfall placement}

Figure 2 and table 4 present the variability of footfall placement during the last 10 steps of the approach run in both testing groups before and after the training programme. The analysis revealed a significant Group $\mathrm{x}$ Time $\mathrm{x}$ Steps interaction $\left(\mathrm{F}_{10,340}\right.$ $=2.57 ; \mathrm{p}=0.007)$. We also found a significant main effect of Group $\mathrm{x}$ Time $\left(\mathrm{F}_{10,190}=4.20 ; \mathrm{p}=0.043\right)$ and effect of Group $\mathrm{x}$ Steps $\left(\mathrm{F}_{119}=9.35 ; \mathrm{p}=0.0087\right)$. It was observed that the variability of footfall placement after the training programme in the EXP group was significantly $(\mathrm{p}<0.01)$ lower than before the programme from the first step analysed until the last three steps before the take-off board.

\section{Step velocity in the approach run}

Figure 3 presents the velocity at a given support phase during the last 10 steps of the approach run in both testing groups before and after the training programme. The analysis revealed a significant Group $\mathrm{x}$ Time $\mathrm{x}$ Steps interaction $\left(\mathrm{F}_{10,340}=1.91\right.$; $\mathrm{p}$

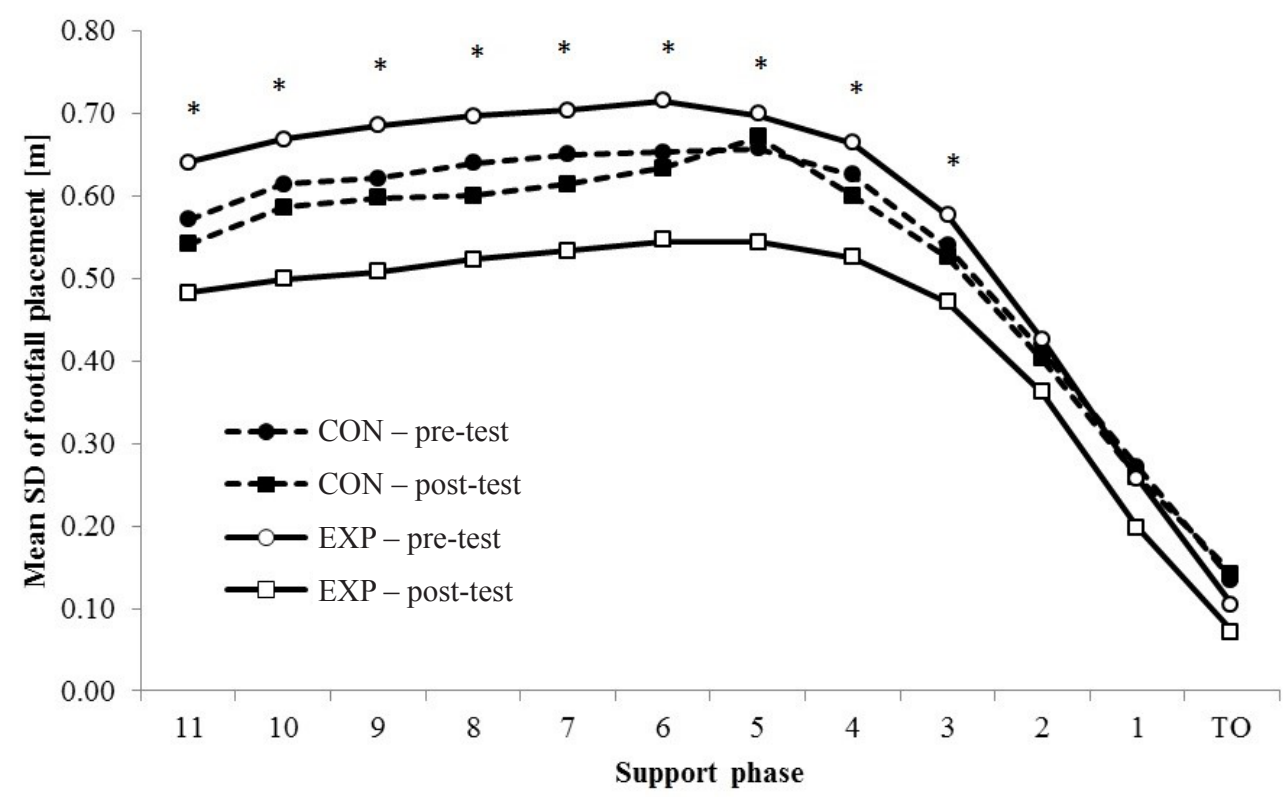

$\mathrm{TO}=$ take-off; * = significantly different from pre-test to post-test in EXP group $(\mathrm{p}<0.01)$

Figure 2. Pre-test and post-test footfall variability during the last 11 support phases of the approach run for the experimental and control groups 
$=0.032)$. There were no significant effects of either Group $\mathrm{x}$ Steps $\left(\mathrm{F}_{10,190}=0.32 ; \mathrm{p}=0.97\right)$ or Group $\mathrm{x}$ Time $\left(\mathrm{F}_{1,34}=1.75 ; \mathrm{p}=\right.$ 0.19). The post-hoc analysis showed that participants in the EXP group increased their step velocity in the last five support phases before the take-off board after the training programme.

\section{Percentage distribution of step length}

Figure 4 presents the percentage distribution of step length in the last 3 steps of the approach run in both testing groups before and after the training programme. There were no significant effects for Group x Time $\left(\mathrm{F}_{1,34}=1.78 ; \mathrm{p}=0.19\right)$, Group $\mathrm{x}$ Steps $\left(\mathrm{F}_{2,68}=0.30 ; \mathrm{p}=0.74\right)$, or Group $\mathrm{x}$ Time $\mathrm{x}$ Steps $\left(\mathrm{F}_{2,68}=0.20 ; \mathrm{p}=\right.$ 0.82 ) interactions.

The analysis showed no significant $(p>0.05)$ differences in the proportions of step length during the last two steps. The subjects from the EXP group displayed mean values of $1.81 \mathrm{~m}$ and $1.85 \mathrm{~m}$ in the pre-test and $1.82 \mathrm{~m}$ and $1.81 \mathrm{~m}$ in the post-test for the penultimate and last step before take-off, respectively.

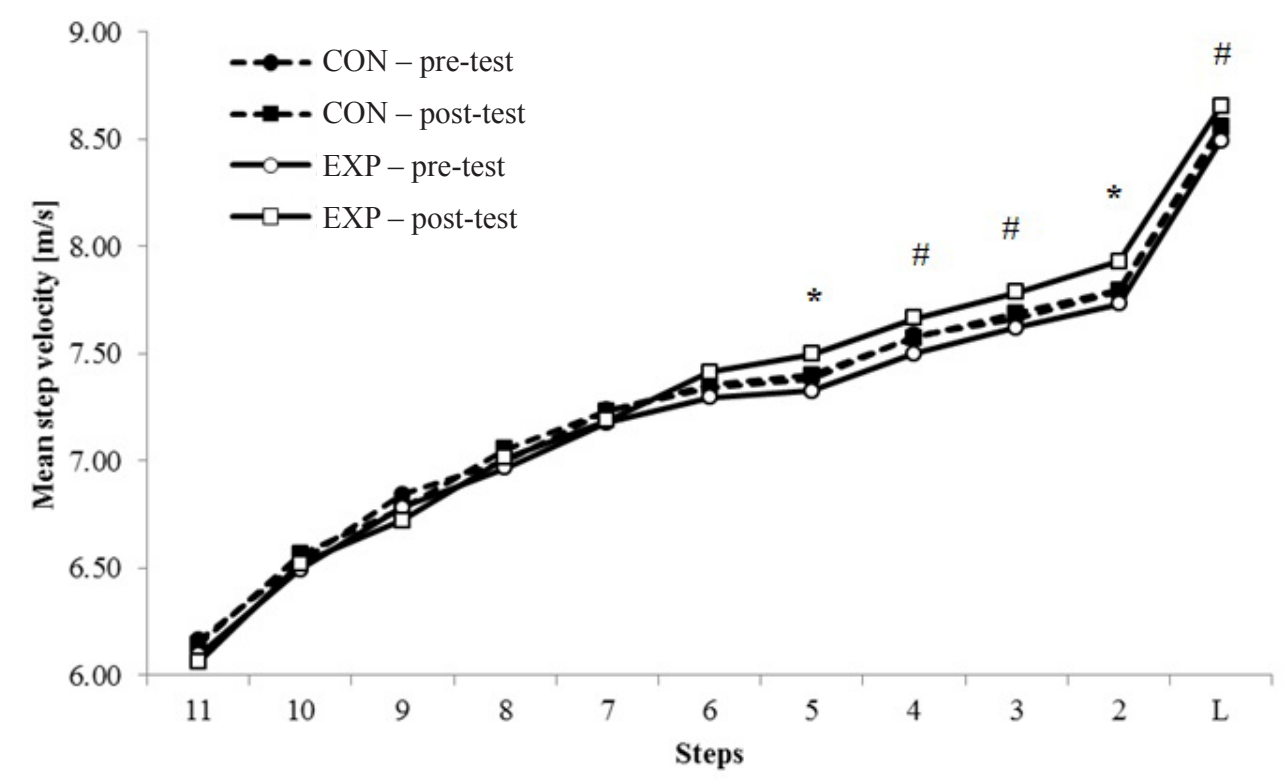

$\mathrm{L}=$ last step before take-off; * $=$ significantly different from pre-test to post-test in EXP group $(\mathrm{p}<0.01)$; \# = significantly different from pre-test to post-test in EXP group ( $\mathrm{p}<0.05)$.

Figure 3. Pre-test and post-test horizontal velocity in the last 11 support phases of the approach run for the experimental and control groups

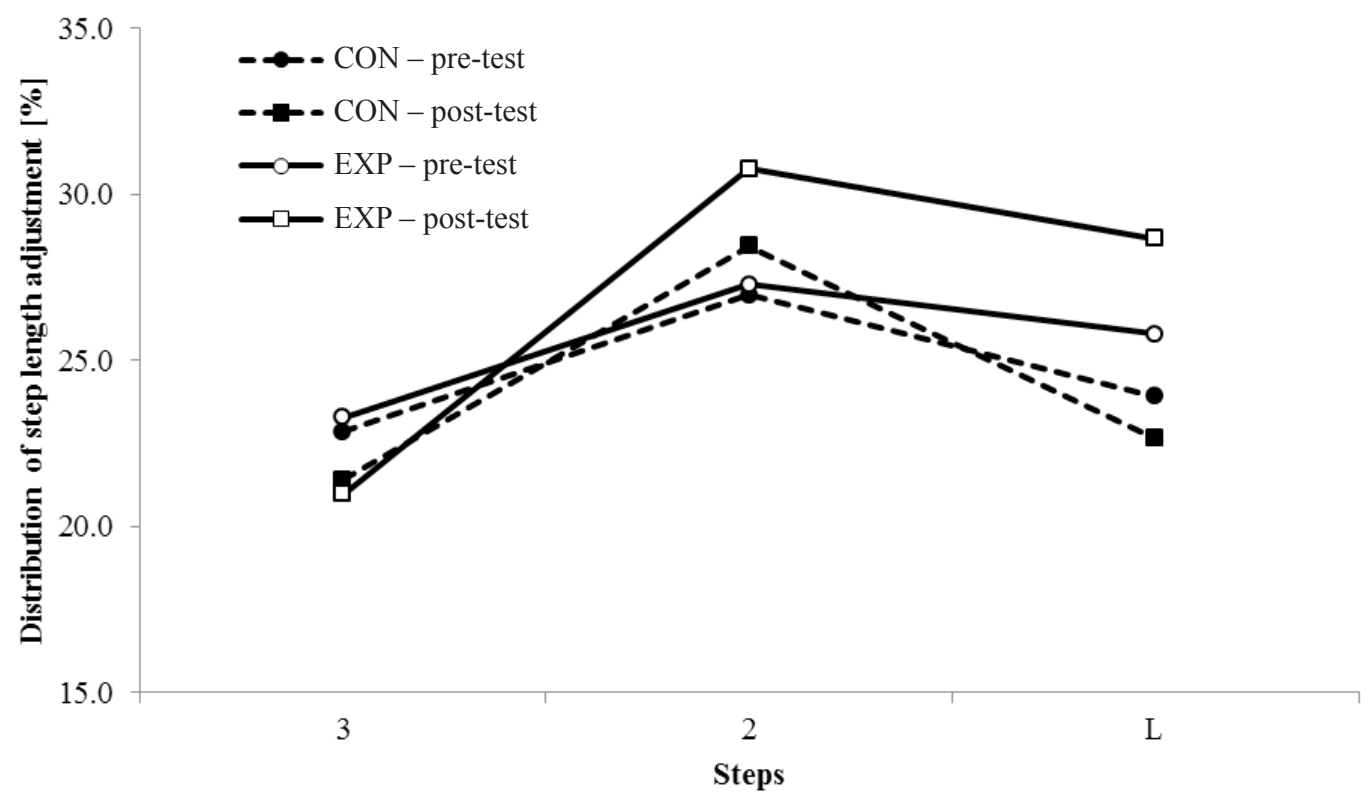

$\mathrm{L}=$ last step before take-off.

Figure 4. Pre-test and post-test percentage distribution of step length adjustment in the last 3 steps of the approach run for the experimental and control groups 
Distance jumped and accuracy at the take-off board

Table 3 shows the results for the distance jumped and accuracy at take-off as means $( \pm \mathrm{SD})$ in the two groups. The results of ANOVA revealed a significant Group $\mathrm{x}$ Time interaction $\left(\mathrm{F}_{1,34}=\right.$ $11.14 ; \mathrm{p}=0.0071)$. The post-hoc analysis showed that subjects in the experimental group jumped significantly $(\mathrm{p}<0.001)$ further after the training programme.

A significant Group $\mathrm{x}$ Time interaction for accuracy at takeoff $\left(\mathrm{F}_{2,28}=7.33, \mathrm{p}=0.044\right)$ was found. The Tukey analysis revealed that participants from the EXP group were more accurate compared to participants from the CON group. It was also found that subjects from the EXP group were significantly $(\mathrm{p}<0.05)$ more accurate after the training programme.
As was the case in previous investigations [21, 26], the analysis of inter-trial footfall variability showed an ascendingdescending trend in both the EXP and CON groups (fig. 2). The amount of footfall variability for both groups after the first testing session was close to that previously reported for non-long jumpers [14, 17]. Moreover, the maximum SD of the TBD did not change from pre-test to post-test and was observed at the $6^{\text {th }}$ and $5^{\text {th }}$ support phases for the EXP and CON groups, respectively. Participants from the EXP group significantly lowered their footfall variability in the first phase of the approach run. A significant decrease was also found in the maximum value of the SD of the toe-to-board distance (tab. 4). Such changes were not observed in the CON group. This may indicate that the training process had a positive impact on establishing a stable move-

Table 3. Pre-test and post-test mean values $( \pm$ SD) for distance jumped and accuracy of take-off for the experimental and control groups

\begin{tabular}{|c|c|c|c|c|}
\hline \multirow{2}{*}{ Group } & \multicolumn{2}{|c|}{ Distance jumped [m] } & \multicolumn{2}{|c|}{ Accuracy of take-off [m] } \\
\cline { 2 - 5 } & Pre-test & Post-test & Pre-test & $0.15 \pm 0.06$ \\
\hline $\operatorname{EXP}(n=20)$ & $4.52 \pm 0.56$ & $4.72 \pm 0.47^{*}$ & $0.11 \pm 0.06^{\sharp \#}$ \\
\hline $\operatorname{CON}(n=16)$ & $4.65 \pm 0.31$ & $4.69 \pm 0.34$ & 0.03 & $0.16 \pm 0.04$ \\
\hline
\end{tabular}

$\mathrm{CON}=$ control group; EXP = experimental group; * = significantly different from pre-test $(\mathrm{p}<0.001)$; $\#=$ significantly different from pre-test $(\mathrm{p}<0.01) ; ¥=$ significantly different from CON group $(\mathrm{p}<0.01)$.

\section{Maximum value of toe-to-board distance $S D$}

Table 4 shows the maximum value of the SD of the toe-toboard distance from pre-test to post-test in the EXP and CON groups. The analysis revealed a significant main effect for Group $\mathrm{x}$ Time $\left(\mathrm{F}_{1,34}=6.85 ; \mathrm{p}=0.0132\right)$. The post-hoc analysis showed that the subjects from the EXP group significantly $(\mathrm{p}<0.01)$ lowered the maximum value of the SD of the toe-board distance after the training programme.

Table 4. Pre-test and post-test maximum values of toe-to-board distance SD for the experimental and control groups

\begin{tabular}{|c|c|c|}
\hline \multirow{2}{*}{ Group } & \multicolumn{2}{|c|}{ Maximum value of toe-to-board distance SD } \\
\cline { 2 - 3 } & Pre-test & Post-test \\
\hline $\operatorname{EXP}(n=20)$ & 71.6 & $54.5^{\star}$ \\
\hline $\operatorname{CON}(n=16)$ & 65.7 & 67.0 \\
\hline
\end{tabular}

$\mathrm{CON}=$ control group; EXP = experimental group; ${ }^{*}=$ significantly different from pre-test $(\mathrm{p}<0.01)$.

\section{Discussion}

The primary goal of this experiment was to investigate how a training programme focusing on increasing consistency in the step pattern would affect the variability of footfall placement during the approach run in the long jump. The secondary objective of the study was to evaluate whether changes in movement variability would influence the performance of the long jump. The results showed that movement variability determined using footfall variability during the approach run in the long jump did change with practice. Subjects from the EXP group significantly increased their footfall consistency in the acceleration phase of the approach run. However, significant differences were not observed during the last three steps before take-off. Moreover, a significant improvement was observed in take-off accuracy as well as velocity during the final part of the approach run. ment pattern during the acceleration phase. This assumption is supported by Seifert et al. [27] who noted that stability, which comes with experience, is a natural tendency of the movement system towards more economical behaviour. Probably, these changes also increased subjects' adaptability to the performance of the approach run, so they were simultaneously able to produce more stable steps in the acceleration phase and remain flexible during the zeroing-in phase. Moreover, these changes are in line with previous findings which emphasise that the reduction in movement variability is related to an increase in expertise [6]. This may also indicate that participants achieved a more stable pattern of behaviour common for the second stage of the motor learning process [28]. According to this concept, at the first stage of learning, individuals attempt to establish an appropriate relationship among motor system components, while at the second stage, learners are able to control the process of movement coordination. This could have led to a reduction in footfall variability and resulted in higher consistency in the performance of the approach run. In light of the present findings, it seems that it is possible to decrease footfall variability in the acceleration phase of the approach run by implementing the training protocol proposed in this study. Nevertheless, the last part of the approach run cannot be directly influenced: the participants from the EXP group spread their footfalls in the final three steps before the take-off board in a similar manner during both testing sessions. On the other hand, they produced lower, though not significantly, amounts of footfall variability. These findings are also supported by the percentage distribution of step length adjustment, where there was no significant difference between testing sessions for the two groups (fig. 4). However, a major part of the overall adjustments were made during the last three support phases before take-off.

The results discussed above prove that the participants in the EXP group moved within a dynamical system framework and had to confront performance constraints. This would indicate that movement variability operates in a functional manner to enable the performance of action $[9,27]$. Another explana- 
tion might be the fact that this is connected with the control mechanism which emerges in the final stage of the approach phase and which probably operated in the same way across the two testing sessions. This mechanism based on perception-action coupling leads to a better interaction with the constraints of action in a dynamical system, and it occurs unless adjustments are required [29]. Thus, in one way, the similar environmental conditions in the two testing sessions could have resulted in no significant differences in footfall variability in the last steps. On the other hand, a decrease in variability during the acceleration phase might have led to a lesser need to make large adjustments over the final steps. It could have also been beneficial for developing a more repeatable step pattern and, as a consequence, resulted in better take-off accuracy (tab. 3). This observation is consistent with Makaruk et al. [21] who stated that lower levels of footfall variability during the execution of the approach run could be linked to better accuracy at the takeoff board. Despite the lack of clear evidence of the influence of accuracy at the take-off board on the performance of the jump (i.e. jumping distance), we believe that reducing the number of foul jumps can increase the likelihood of better performance. For example, from a practical point of view, any valid attempts made by the participants of a jumping competition may result in them qualifying to the final round and determine their place in the competition.

The post-test results for approach run velocity also revealed differences in the EXP group (fig. 3). The participants significantly increased their approach run velocity; however, this change was observed only during the final stage of the approach run (from $7.30-8.50 \mathrm{~m} / \mathrm{s}$ for the pre-test to $7.41-8.66 \mathrm{~m} / \mathrm{s}$ for the post-test from the $5^{\text {th }}$-to-last step). It must be highlighted that these findings could have been expected as the design of the experimental programme included exercises that could influence the participants' speed capabilities. Interestingly, in the first phase of the run-up, the subjects did not significantly increase their step velocity despite the improvement in step variability. Nevertheless, differences were observed in the final steps before take-off, when they displayed similar footfall variability as they did before the training was applied. We supposed that through the improvement in step precision they established a more repeatable set of movements. The possible explanation for this finding may be linked with the stabilisation of the spatial and temporal components (i.e. step length or step frequency) which must have a crucial influence on step velocity. This step consistency could provide a stable basis for increasing speed at the end of the approach run. Therefore, stabilisation in the locomotor pattern resulted in the fact that participants could focus on maintaining horizontal velocity and an appropriate running rhythm. Furthermore, these results are worth considering in light of the speed-accuracy trade-off [30]. With the significant improvement in the take-off velocity, participants from the EXP group were at the same time significantly more accurate at the take-off board.

Additionally, as we had expected, participants from the EXP group significantly improved the distance jumped (tab. 3). This result could be related to increased horizontal velocity at takeoff, as it strongly influences the jumping distance [31]. However, we suppose that this improvement in the jumping distance may be connected with an improvement in the locomotor pattern, which could have led to better take-off technique. Probably, it was also due to certain changes which occurred during the execution of the last part of the approach run. The proportions of the penultimate to the last step demonstrated by participants from the EXP group differed, although not significantly, from pre-test to post-test. They changed the step proportions from the less favourable pattern of lengthening the last step to a more beneficial pattern of shortening the last step, which is commonly observable in the preparation for take-off in horizontal jumps. This could have improved the position of the centre of the mass and might have caused positive changes in the other crucial variables [31]. Future investigations should be focused on seeking relations between movement variability and crucial kinematics of the jump.

There are some limitations to this study. Firstly, the subjects were non-long jumpers without experience in long jump training, so it cannot be unequivocally concluded that similar changes can be observed in athletes with a higher level of experience. Secondly, it is worth mentioning that the analysis did not include the initial 4-6 steps of the approach run. However, based on previous investigations $[14,25]$, we believe that an analysis of step variability during this part of the approach run would not significantly change the overall view the subjects' regulation found in this study. Finally, the findings from this investigation should be interpreted cautiously, as the subjects were grouped together, and some effects on changing movement patterns could have been masked [32]. Future research should involve inter-individual analyses of movement variability during the approach run and its influence on crucial kinematic parameters which are required for a successful jump. Additionally, in light of the findings of some studies [33, 34], training routines should not only concern developing perfect regularity and a stereotype step pattern, but they should also enhance the adaptability of an athlete to the constraints of the action. Therefore, future research should investigate the effect of training which focuses on increasing individual interactions with the dynamic environment in the process of learning how to perform the approach run.

\section{Conclusions}

The results of this study suggest that training targeted at improving step consistency and not only on developing a repeatable approach run makes it possible to establish more stable movement behaviour in the acceleration phase of the long jump. Moreover, the lack of differences in footfall variability in the final phase of the approach run emphasises the functional nature of movement variability within the dynamic environment. Finally, we found that decreased footfall variability during the acceleration phase in the long jump approach run also has a positive effect on take-off accuracy and velocity during the final steps.

\section{Acknowledgements}

This research was supported by the Polish Ministry of Science and Higher Education under the project "Development of Academic Sport” (No. RSA2 03452). The experimental data were gathered in the Laboratory of Biomechanics and Kinesiology and Laboratory Diagnostics Comprehensive Agreement on Human Movement and Exercise Capacity in the Regional Centre of Research and Development in Biała Podlaska co-financed under the European Regional Development Fund, Operational Programme Development of Eastern Poland 2007-2013. 


\section{Literature}

1. Stergiou N., Decker L.M. (2011). Human movement variability, nonlinear dynamics, and pathology: Is there a connection? Human Movement Science 30(5), 869-888.

2. Newell K.M., Corcos D.M. (1993). Variability and motor control. Champaign: Human Kinetics.

3. Newell K.M. (1986). Constraints on the development of coordination. In M.G. Wade, H.T.A. Whiting (eds), Motor development in children. Aspects of coordination and control (pp. 341-360). Dordrecht, Germany: Martinus Nighoff.

4. Bartlett R., Wheat J., Robins M. (2007). Is movement variability important for sport biomechanists? Sports Biomechanics 6(2), 224-243.

5. Glazier P.S., Davids K.W. (2009). On analysing and interpreting variability in motor output. Journal of Science and Medicine in Sport 12(4), e2-e3.

6. Stergiou N., Harbourne R.T., Cavanaugh J.T. (2006). Optimal movement variability: A new theoretical perspective for neurologic physical therapy. Journal of Neurologic Physical Therapy 30, 120-129.

7. Bradshaw E.J., Keogh J.W.L., Hume P.A., Maulder P., Nortje J., Marnewick M.(2009). The effect of biological movement variability on the performance of the golf swing in high- and low-handicapped players. Research Quarterly for Exercise and Sport 80(2), 185-196.

8. Button C., MacLeod M., Sanders R., Coleman S. (2003). Examining movement variability in the basketball freethrow action at different skill levels. Research Quarterly for Exercise and Sport 74, 257-269.

9. Davids K., Glazier P., Araujo D., Bartlett R. (2003). Movement systems as dynamical systems: the functional role of variability and its implications for sports medicine. Sports Medicine 33, 245-260.

10. Bartlett R.M. (2008). Movement variability and its implications for sports scientists and practitioners: An overview. International Journal of Sports Science E Coaching 1(3), 113124.

11. Chow J.Y., Davids K., Button C., Koh M. (2007). Variation in coordination of a discrete multiarticular action as a function of skill level. Journal of Motor Behavior 39, 463-479.

12. Wilson C., Simpson S.E., van Emmerik R.E., Hamill J. (2008). Coordination variability and skill development in expert triple jumpers. Sports Biomechanics 7(1), 2-9.

13. Lee D.N., Lishman J.R., Thomson J.A. (1982). Regulation of gait in long jumping. Journal of Experimental Psychology: Human Perception and Performance 8, 448-459.

14. Scott M.A., Li F., Davids K. (1997). Expertise and the regulation of gait in the approach phase of the long jump. Journal of Sports Sciences 15, 597-605.

15. Hay J.G. (1988). Approach strategies in the long jump. International Journal of Sport Biomechanics 4, 114-129.

16. Panteli F., Smirniotou A., Theodorou A. (2016). Performance environment and nested task constraints influence long jump approach run: a preliminary study. Journal of Sports Sciences 34(12), 1116-1123.

17. Starzak M., Makaruk H., Sadowski J. (2015). Step length adjustment in the long jump with and without take-off board in non-long jumpers. Antropomotoryka. Journal of Kinesiology and Exercise Sciences 70(25), 19-25.
18. Theodorou A., Ioakimidou E., Kouris P., Panoutsakopoulos V., Smpokos E. (2013). Colour contrast and regulation of the long jump approach run. Journal of Human Sport E Exercise 8(3), 681-687.

19. Greenwood D.A. (2014). Informational constraints on performance of dynamic interceptive actions. Doctoral thesis, Queensland University of Technology, Brisbane, Australia.

20. Makaruk H., Starzak M., Lopez J.L. (2015). The role of a check-mark in step length adjustment in long jump. Journal of Human Sport and Exercise 10(3), 756-763.

21. Makaruk H., Starzak M., Sadowski J. (2015). Does step length adjustment determine accuracy of take-off and approach run velocity in long and triple jumps? Human Movement 16(3), 124-129.

22. Lundin P., Berg W. (1993). Developing the approach in the jumps. New Studies of Athletic 8(1), 45-50.

23. Galloway M., Connor K. (1999). The effect of steering on stride pattern and velocity in long jump. Scientific proceedings "XVII International Symposium on Biomechanics in Sports” (pp. 41-44). Perth, Western Australia: Edith Cowan University.

24. Makaruk B., Makaruk H., Sacewicz T. (2009). The efficacy of speed training conducted by applying runs between guide strips. Wychowanie Fizyczne i Sport 53(3), 167-172.

25. Veligekas P., Bogdanis G.C., Stabolitis C., Frantzis D. (2012). Rest interval between jumps during track and field jumping competitions. Book of Abstracts of the $17^{\text {th }}$ Annual Congress of the European College of Sport Science (pp. 604). Bruges, Belgium: E.C.S.S.

26. Hay J.G., Koh T.J. (1988). Evaluating the approach in horizontal jumps. International Journal of Sport Biomechanics 4, 372-392.

27. Seifert L., Button C., Davids K. (2013). Key properties of expert movement systems in sport: An ecological dynamics perspective. Sports Medicine 43, 167-178.

28. Newell K.M. (1985). Coordination, control and skill. In D. Goodman, R.B. Wilberg, I.M. Franks (eds), Differing perspectives in motor learning, memory, and control (pp. 295317). Amsterdam: Elsevier.

29. Montagne G., Cornus S., Glize D., Quaine F., Laurent M. (2000). A perception-action coupling type of control in long jumping. Journal of Motor Behavior 32, 37-43.

30. Fitts P.M. (1954). The information capacity of the human motor system in controlling the amplitude of movement. Journal of Experimental Psychology 47, 381-391.

31. Lees A., Graham-Smith P., Fowler N. (1994). A biomechanical analysis of the last stride, touchdown, and takeoff characteristics of the men's long jump. Journal of Applied Biomechanics 10, 61-78.

32. MacPherson A.C., Collins D., Graham-Smith P., Turner A.P. (2013). Using rhythmicity to promote performance in horizontal jumps: An exemplar of the need for intra-individual intervention. International Journal of Sport Psychology 44, 93-110.

33. Bradshaw E.J., Aisbett B. (2006). Visual guidance during competition performance and run-through training in long jumping. Sports Biomechanics 5(1), 1-14.

34. Glize D., Laurent M. (1997). Controlling locomotion during the acceleration phase in sprinting and long jumping. Journal of Sports Sciences 15, 181-189.

Submitted: June 10, 2016

Accepted: September 20, 2016 